\title{
Controlling physical systems with symmetries
}

\author{
R. O. Grigoriev and M. C. Cross \\ Condensed Matter Physics 114-36, California Institute of Technology, Pasadena, California 91125
}

(Received 22 July 1997)

\begin{abstract}
Symmetry properties of the evolution equation and the state to be controlled are shown to determine the basic features of the linear control of unstable orbits. In particular the selection of control parameters and their minimal number are determined by the irreducible representations of the symmetry group of the linearization about the orbit to be controlled. We use the general results to demonstrate the effect of symmetry on the control of two sample physical systems: a coupled map lattice and a particle in a symmetric potential.
\end{abstract}

[S1063-651X(98)04302-5]

PACS number(s): 05.45.+b, 07.05.Dz, 11.30.Na

\section{INTRODUCTION}

Despite the recent wave of interest towards controlling chaotic dynamics [1,2], an interesting and important question of controlling systems with symmetries received surprisingly little attention in the physics literature. The importance of symmetries in controlling, for instance, spatiotemporal chaos is evident since the systems typically show rotational and translational symmetries. Although the presence of symmetries usually significantly simplifies the analysis of system dynamics, it also makes control schemes more complicated due to the inherent degeneracies of the evolution operators. In fact, the presence of symmetries, explicit or implicit, makes a number of single-control-parameter methods fail [1,3], calling for multiparameter control [4-7].

In order to see how these restrictions arise, let us consider a general discrete-time system (the arguments for continuous-time systems are very similar), whose evolution is described by the map $F: \mathbb{R}^{N} \times \mathrm{R}^{M} \rightarrow \mathbb{R}^{N}$,

$$
\mathbf{z}^{t+1}=F\left(\mathbf{z}^{t}, \mathbf{p}\right),
$$

where $\mathbf{z}$ is an $N$-dimensional state vector and $\mathbf{p}$ is an $M$-dimensional parameter vector. Linearizing about the steady-state solution of $\mathbf{z}^{*}=F\left(\mathbf{z}^{*}, \mathbf{p}^{*}\right)$ and denoting $\mathbf{x}^{t}=\mathbf{z}^{t}$ $-\mathbf{z}^{*}$ and $\mathbf{u}^{t}=\mathbf{p}^{t}-\mathbf{p}^{*}$, we readily obtain

$$
\mathbf{x}^{t+1}=A \mathbf{x}^{t}+B \mathbf{u}^{t}
$$

where $A_{i j}=\partial F_{i}\left(\mathbf{z}^{*}, \mathbf{p}^{*}\right) / \partial z_{j}$ is the Jacobian of the transformation and $B_{i j}=\partial F_{i}\left(\mathbf{z}^{*}, \mathbf{p}^{*}\right) / \partial p_{j}$ is the control matrix.

If the steady state $\mathbf{z}^{*}$ is unstable, it can be stabilized by an appropriate feedback through the time-dependent control perturbation $\mathbf{u}^{t}$ if the matrices $A$ and $B$ satisfy certain conditions. We will understand the design of the control scheme as an appropriate choice of the control matrix $B$. We will see below that the conditions affecting this choice can be easily obtained from the symmetry properties of the system and the controlled state.

\section{STABILIZABLE VS CONTROLLABLE SYSTEMS}

Assuming that the feedback is linear in the deviation from the steady state $\mathbf{z}^{*}$, we can write

$$
\mathbf{u}^{t}=-K \mathbf{x}^{t}
$$

and one obtains the linearized evolution equation in the form

$$
\mathbf{x}^{t+1}=(A-B K) \mathbf{x}^{t} .
$$

The matrix $A^{\prime}=A-B K$ has stability properties different from the stability properties of the matrix $A$. This can be exploited to make the steady state $\mathbf{z}^{*}$ (the matrix $A-B K$ ) stable under control.

The dynamical system (2) or the pair $(A, B)$ is said to be stabilizable if there exists a state feedback (3) such that the system (4) is stable. Stabilizability is a property that often depends sensitively on the values of the control parameters $\mathbf{p}^{*}$.

In the majority of practical situations it is preferable to have an adaptive control that would stabilize a given steady state $\mathbf{z}^{*}\left(\mathbf{p}^{*}\right)$ for arbitrary values of the system parameters. This is especially important if one is to track the trajectory $\mathbf{z}^{*}$ as $\mathbf{p}^{*}$ changes or use the same control arrangement to stabilize different steady (or even periodic) states.

Such a control scheme is obtained if the more restrictive condition of controllability is imposed on the matrices $A$ and $B$. The dynamical system (2) or the pair $(A, B)$ is said to be controllable if the eigenvalues of the matrix $A-B K$ can be freely assigned (with complex ones in conjugate pairs), which is equivalent (see Theorem 5.13 in Ref. [8]) to requiring that:

$$
\operatorname{rank}(C)=N,
$$

where $C=\left(B A B A^{2} B \cdots A^{N-1} B\right)$ is called the controllability matrix. Relation (5) was introduced into the physics literature from linear systems theory by Romeiras et al. [1] as a simple but practical test of the controllability.

In order to better understand the restrictions imposed by the symmetry, it is beneficial to look at the controllability condition written in the form (5) from the geometrical point of view assuming $M=1$ and $B=\mathbf{b}$. Suppose we let the system evolve under control for $\tau$ steps from the initial state $\mathbf{x}^{t}$. The final state will be given by

$$
\mathbf{x}^{t+\tau}=A^{\tau} \mathbf{x}^{t}+\sum_{m=0}^{\tau-1} A^{\tau-1-m} \mathbf{b} u^{t+m} .
$$


The controllability in this context is equivalent to the vectors

$$
\mathbf{f}^{m}=A^{\tau-1-m} \mathbf{b}, \quad m=0, \ldots, \tau-1
$$

spanning the state space for $\tau=N$, so that any initial state can be mapped to any final state in $\tau$ time steps by an appropriate choice of the "coordinates" $u^{t+m}$ of the difference $\mathbf{x}^{t+\tau}-A^{\tau} \mathbf{x}^{t}$

If the matrix $A$ is nondegenerate (has a nondegenerate spectrum), one can always find a vector $\mathbf{b}$ such that the resulting set (7) forms a basis. However, if $A$ is degenerate (which is a usual consequence of symmetry), there will exist an invariant subspace $L^{r} \subset \mathbb{R}^{N}$, with the dimension $\operatorname{dim}\left(L^{r}\right)$ $>1$, where the dynamics of the system cannot be controlled with just one control parameter (see Ref. [1] for an example of such a situation).

If the system dynamics in $L^{r}$ happens to be stable, the system can still be stabilized similarly to the nondegenerate case, but we have to ensure controllability in case the dynamics in this subspace is unstable. This can be achieved by increasing the number of control parameters $M$, which extends the set (7), until it spans every unstable invariant subspace of $\mathrm{R}^{N}$. This would lead one to assume that $M$ should be defined by the dimension of the largest invariant subspace or equivalently, the highest degeneracy of the Jacobian ma$\operatorname{trix} A$. We will see, however, that various kinds of degeneracy have a somewhat different effect on the controllability of the system.

\section{THE NUMBER AND SELECTION OF PARAMETERS}

Let us assume that the evolution equation (1) possesses a symmetry described by a symmetry group $\mathcal{G}$, i.e., the map $F$ commutes with all group actions

$$
F(g(\mathbf{z}), \mathbf{p})=g(F(\mathbf{z}, \mathbf{p})) \quad \forall g \in \mathcal{G}
$$

or in other words, the function $F(\mathbf{z}, \mathbf{p})$ is $\mathcal{G}$ equivariant. The symmetry of the linearized equation (2) in the absence of control $(\mathbf{u}=\mathbf{0})$ is in general different from (although closely related to) the symmetry of the full nonlinear equation (1). We will call the respective symmetry group $\mathcal{G}^{*}$. It generates the matrix representation $T$ in the state space $\mathbb{R}^{N}$ :

$$
[g(\mathbf{x})]_{i}=[T(g) \mathbf{x}]_{i}=T(g)_{i j} x_{j}
$$

Due to the symmetry, all matrices $T(g)$ commute with the Jacobian

$$
T(g) A=A T(g) \quad \forall g \in \mathcal{G}^{*} .
$$

$\mathcal{G}^{*}$ depends on both $\mathcal{G}$ and the reference state $\mathbf{z}^{*}$ or, to be exact, its symmetry group, which we denote $\mathcal{H}$ :

$$
h\left(\mathbf{z}^{*}\right)=\mathbf{z}^{*} \quad \forall h \in \mathcal{H} .
$$

The symmetry of the evolution equation is reduced upon linearization, if the reference state has low symmetry, and then $\mathcal{G}^{*}$ becomes one of the subgroups of $\mathcal{G}$. On the other hand, $\mathcal{G}^{*}$ might be equal to $\mathcal{G}$ or even include $\mathcal{G}$ as a subgroup for highly symmetric reference states, with the apparent symmetry increased by linearization.
Decomposing $T$ into a sum of irreducible representations $T^{r}$ of the group $\mathcal{G}^{*}$ with respective dimensionalities $m_{r}, \quad r=1, \ldots, l$ we obtain

$$
T=\sum_{r \oplus} T^{r}, \quad N=\sum_{r} m_{r}
$$

According to the standard group-theoretic analysis [9], the Jacobian $A$ will have eigenvalues $\lambda_{r}$ with multiplicities $n_{r}$ $\geqslant m_{r}$, corresponding to the dimensions of the irreducible representations $T^{r}$ contained in the decomposition of $T$. If $m_{r}>1$ for some $r$, the Jacobian becomes degenerate, which causes certain control methods to fail (see, for example, Ref. [3]).

Next we use the result of linear systems theory based on the Jordan decomposition of the Jacobian matrix

$$
\Lambda=\operatorname{SAS}^{-1}=\left(\begin{array}{cccc}
\Lambda^{1} & & & \\
& \Lambda^{2} & & \\
& & \ddots & \\
& & & \Lambda^{l}
\end{array}\right),
$$

where the Jordan superblock

$$
\Lambda^{r}=\left(\begin{array}{cccc}
\Lambda^{r 1} & & & \\
& \Lambda^{r 2} & & \\
& & \ddots & \\
& & & \Lambda^{r s_{r}}
\end{array}\right)
$$

corresponding to the eigenvalue $\lambda_{r}$ has dimension $n_{r}$ and consists of $s_{r}$ Jordan blocks

$$
\Lambda^{r i}=\left(\begin{array}{ccccc}
\lambda_{r} & & & & \\
1 & \lambda_{r} & & & \\
& \ddots & & & \\
& & 1 & \lambda_{r} & \\
& & & 1 & \lambda_{r}
\end{array}\right) .
$$

Since the controllability is invariant with respect to coordinate transformations (Theorem 5.17 in Ref. [8]), condition (5) is satisfied for the pair $(A, B)$ if and only if it is satisfied for the pair $(\Lambda, \hat{B})$, where $\hat{B}=S B$ is the transformed control matrix.

If $\hat{B}$ is partitioned according to the block structure of $\Lambda$,

$$
\hat{B}=\left(\begin{array}{c}
\hat{B}^{1} \\
\hat{B}^{2} \\
\vdots \\
\hat{B}^{l}
\end{array}\right), \quad \hat{B}^{r}=\left(\begin{array}{l}
\hat{B}^{r 1} \\
\hat{B}^{r 2} \\
\vdots \\
\hat{B}^{r s_{r}}
\end{array}\right), \quad \hat{B}^{r i}=\left(\begin{array}{c}
\hat{b}_{1}^{r i} \\
\hat{b}_{2}^{r i} \\
\vdots \\
\hat{b}_{n_{r i}}^{r i}
\end{array}\right),
$$

the controllability condition for the pair $(\Lambda, \hat{B})$ is reduced to the controllability conditions for each pair $\left(\Lambda^{r}, \hat{B}^{r}\right)$, which, in turn, is satisfied (Theorem 5.18 in Ref. [8]) if and only if for each $r$ the set of $s_{r} M$-dimensional row vectors

$$
\hat{b}_{1}^{r 1}, \hat{b}_{1}^{r 2}, \ldots, \hat{b}_{1}^{r s_{r}}
$$


is linearly independent. This in turn can be achieved if and only if $M \geqslant s_{r}$ for every $r$. Hence the minimal number of control parameters should equal the maximal number of Jordan blocks contained in any one superblock

$$
M_{\min }=\max _{r} s_{r} .
$$

In general, the system under consideration is not Hamiltonian and therefore its Jacobian matrix $A$ is not Hermitian and hence nondiagonalizable. Therefore, we have $n_{r} \geqslant s_{r}$ $\geqslant m_{r}$. However, in the absence of accidental degeneracies $n_{r}=s_{r}=m_{r}$ for every $r$ and the condition (17) is equivalent to

$$
\operatorname{rank}\left(\hat{B}_{r}\right)=m_{r} .
$$

The calculation of the transformation $S$ can be avoided for compact groups $\mathcal{G}^{*}$ by using the projection operator $P^{r}$ on the subspace $L^{r} \subset \mathrm{R}^{N}$, which transforms according to the $r$ th irreducible representation

$$
P^{r}=m_{r} \int_{\mathcal{G}^{*}} \chi^{r}(g) T(g) d \mu(g) .
$$

Here $\chi^{r}(g)$ is the character of the group element $g$ in the representation $T^{r}$ and $d \mu(g)$ is the group measure [9]. For finite groups this integral is replaced with the sum.

Using the fact that

$$
\operatorname{rank}\left(\widetilde{B}^{r}\right)=\operatorname{rank}\left(\hat{B}^{r}\right),
$$

where $\widetilde{B}^{r}=P^{r} B$, we conclude (assuming there are no accidental degeneracies) that the controllability condition is satisfied whenever

$$
M \geqslant \max _{r} m_{r}
$$

and $m_{r}$ of $M$ columns of $\widetilde{B}^{r}$ are linearly independent, i.e., form a basis in the eigenspace $L^{r}$ (columns of $\widetilde{B}^{r}$ span $L^{r}$ ) for every $r$. Therefore, the minimal number of independent control parameters $M_{\min }$ is equal to the dimensionality $m_{r}$ of the largest irreducible representation $T^{r}$ present in the decomposition of the representation $T$ of the group $\mathcal{G}^{*}$ in the state space $\mathbb{R}^{N}$.

Regarding the control matrix $B$ as a row of $M$ vectors

$$
B=\left(\begin{array}{llll}
\mathbf{b}_{1} & \mathbf{b}_{2} & \cdots & \mathbf{b}_{M}
\end{array}\right),
$$

we see that the control scheme yielding a controllable system is obtained by choosing the vectors $\mathbf{b}_{i}$ such that $m_{r}$ of the projections $P^{r} \mathbf{b}_{i}$ would be linearly independent for every $r$.

If accidental degeneracies are present, symmetry properties only give a lower bound on the number of required control parameters and one should look at the Jordan block structure of the Jacobian to determine the controllability using the more general conditions (18) and (17). It is easy to see intuitively why the number of control parameters is determined by the number of the Jordan blocks $s_{r}$ and not the multiplicity $n_{r}$ if we look at the Jacobian already reduced to the Jordan form. For instance,

$$
A_{1}=\left(\begin{array}{lll}
\lambda & & \\
& \lambda & \\
& & \lambda
\end{array}\right)
$$

generates the set of three linearly dependent vectors $\mathbf{f}^{0}$ $=\mathbf{b}, \mathbf{f}^{1}=\lambda \mathbf{b}$, and $\mathbf{f}^{2}=\lambda^{2} \mathbf{b}$ [compare to Eq. (7)], that span the one-dimensional subspace of $R^{3}$ for any choice of $\mathbf{b}$. As a result, three linearly independent vectors $\mathbf{b}_{1}, \mathbf{b}_{2}, \mathbf{b}_{3}$ are necessary to control the system.

On the contrary, the Jacobian

$$
A_{2}=\left(\begin{array}{lll}
\lambda & & \\
1 & \lambda & \\
& 1 & \lambda
\end{array}\right)
$$

generates the linearly independent set of basis vectors that spans $\mathbb{R}^{3}$, requiring just one control vector $\mathbf{b}$.

Finally, we should note that symmetry does not always make the Jacobian degenerate and the nondegenerate case can be handled in the same way as the one with no symmetries. Neither does the degeneracy by itself imply that multiparameter control is required. Even if $n_{r}>0$ for some $r$ $=r^{\prime}$ (there is a degeneracy), but $s_{r}=m_{r}=1$ for every $r$ (the degeneracy is accidental and the Jordan block $\Lambda_{r^{\prime}}$ is not block diagonalizable), one control parameter is indeed sufficient to make the system controllable.

\section{CONTINUOUS-TIME SYSTEMS AND PERIODIC ORBITS}

The obtained results hold for continuous-time systems and can be easily generalized to periodic trajectories. We should first observe that a periodic trajectory of period $\tau$ can be treated as a fixed point solution of the superposition of $\tau$ maps. The equation $\mathbf{z}^{*}=F^{\tau}\left(\mathbf{z}^{*}, \mathbf{p}^{*}\right)$ has $\tau$ solutions corresponding to the points of the periodic orbit $\mathbf{z}_{k}^{*}, k$ $=1, \ldots, \tau$.

Next we define the time-dependent single-step Jacobian

$$
A_{i j}^{k}=\frac{\partial F_{i}\left(\mathbf{z}_{k}^{*}, \mathbf{p}^{*}\right)}{\partial z_{j}}
$$

and the control matrix

$$
B_{i j}^{k}=\frac{\partial F_{i}\left(\mathbf{z}_{k}^{*}, \mathbf{p}^{*}\right)}{\partial p_{j}} .
$$

The controllability condition (5) can be extended to timedependent orbits by requiring that rank $\left(C_{k}\right)=N$, where $C_{k}$ $=\left(B_{k} A_{k} B_{k-1} \cdots A_{k} \cdots A_{k-N+2} B_{k-N+1}\right)$ is a generalization of the controllability matrix, for every $k$. requiring that the pairs $\left(A_{k}, B_{k}\right)$ be controllable for every $k$.

Now suppose that the symmetry of the state $\mathbf{z}_{k}^{*}$ is described by the group $\mathcal{H}_{k}$ such that $\mathcal{H}_{k} \subseteq \mathcal{G}$. We can then write

$$
h\left(\mathbf{z}_{k+1}^{*}\right)=h\left(F\left(\mathbf{z}_{k}^{*}\right)\right)=F\left(h\left(\mathbf{z}_{k}^{*}\right)\right)=F\left(\mathbf{z}_{k}^{*}\right)=\mathbf{z}_{k+1}^{*}
$$

for every $h \in \mathcal{H}_{k}$ or, consequently,

$$
\mathcal{H}_{1} \subseteq \mathcal{H}_{2} \subseteq \cdots \subseteq \mathcal{H}_{\tau} \subseteq \mathcal{H}_{1},
$$


i.e., the symmetry group of all the states of the trajectory is the same and can be determined using $\mathbf{z}_{k}^{*}$ with an arbitrary $k$ : $\mathcal{H}=\mathcal{H}_{k}$.

This in turn means that $\mathcal{G}^{*}$ too is unique for any given periodic trajectory, as is the representation $T$. It is therefore enough to know the symmetry properties of an arbitrary point of the periodic trajectory in order to establish the requirements on the control scheme similarly to the timeindependent case.

Finally, consider a continuous-time evolution equation. It can generally be written as

$$
\partial_{t} \mathbf{z}(t)=F(\mathbf{z}(t), \mathbf{p})
$$

Linearization of Eq. (30) around the steady state $\mathbf{z}^{*}$, similarly to the discrete-time case, yields

$$
\partial_{t} \mathbf{x}(t)=A \mathbf{x}(t)+B \mathbf{u}(t)
$$

and we again denote $\mathcal{G}^{*}$ as the group of all actions commuting with the action of the Jacobian. The controllability of the pair $(A, B)$ ensures that all eigenvalues of $A-B K$ can be chosen to be negative, so that the steady state becomes stable. As a result, the control matrix $B$ should satisfy the same conditions as those obtained for the discrete-time case.

\section{COUPLED MAP LATTICE}

Next we apply the general results to the case of the coupled map lattice defined by the evolution equation

$$
z_{i}^{t+1}=\epsilon f\left(z_{i-1}^{t}\right)+(1-2 \epsilon) f\left(z_{i}^{t}\right)+\epsilon f\left(z_{i+1}^{t}\right),
$$

with $i=1,2, \ldots, N$ and the periodic boundary conditions, i.e., $z_{i+N}^{t}=z_{i}^{t}$, imposed. The local map function $f(x)$ can be chosen arbitrarily.

The symmetry group $\mathcal{G}$ of the lattice includes translations by an integer number of lattice sites (periodic boundary conditions make the group finite) and reflections about any site. The corresponding point group is $\mathrm{C}_{N v}$. It has a total of $N / 2+3$ nonequivalent irreducible representations $T^{r}$ : the first four are one-dimensional, $m_{1}=m_{2}=m_{3}=m_{4}=1$, while the rest $N / 2-1$ are two-dimensional, $m_{r}=2, r \geqslant 5$.

Linearizing Eq. (32) about the steady state $\mathbf{z}^{*}$, we obtain Eq. (2) with $A=C D$, where

$$
C_{i j}=(1-2 \epsilon) \delta_{i, j}+\epsilon\left(\delta_{i, j-1}+\delta_{i, j+1}\right)
$$

(with $\delta_{i, j \pm 1}$ extended to comply with periodic boundary conditions) and

$$
D_{i j}=f^{\prime}\left(z_{i}^{*}\right) \delta_{i, j}
$$

This partition of the Jacobian into the product of two matrices explicitly shows how the symmetry group $\mathcal{G}^{*}$ depends on the symmetries of the nonlinear evolution equation and the controlled state $\mathbf{z}^{*}$. The matrix $C$ has all the symmetries imposed by the chosen intersite couplings of the nonlinear model:

$$
T(g) C=C T(g) \quad \forall g \in \mathcal{G},
$$

while the matrix $D$ reflects the symmetries of the reference state $\mathbf{z}^{*}$ :

$$
T(h) D=D T(h) \quad \forall h \in \mathcal{H},
$$

and since the Jacobian $A$ only commutes with matrices that commute with both $C$ and $D, \mathcal{G}^{*}$ should be a maximal subgroup of $\mathcal{G}$ and $\mathcal{H}$.

Deriving the restrictions on the control matrix $B$ is the next step. Once the group $\mathcal{G}^{*}$ is determined, we construct its $N$-dimensional representation $T$ and decompose it into the sum of the irreducible representations of $\mathrm{C}_{N v}$. For instance, a zigzag state gives $\mathcal{G}^{*}=\mathrm{C}_{n v}$ with $n=N / 2$ and $M=m_{5}=2$; a space-period-s not reflection-invariant state corresponds to $\mathcal{G}^{*}=\mathrm{C}_{n}$ with $n=N / s$ and $M=m_{1}=1$, etc.

In particular a uniform reference state has $\mathcal{G}^{*}=\mathrm{C}_{N v}$ and

$$
T=T^{1} \oplus T^{4} \oplus T^{5} \oplus \cdots \oplus T^{N / 2+3},
$$

where each of the representations $T^{5}$ corresponds to the subspace $L^{k}$, generated by the Fourier modes $\left(\mathbf{e}^{k}\right)_{l}=\exp ( \pm i k l)$ with wave vectors $0<|k|<\pi, \quad T^{1}$ to $k=0$, and $T^{4}$ to $k$ $=\pi$. Since $T^{5}$ is present, $M=m_{5}=2$. Therefore, in order to control an unstable uniform steady state of the coupled map lattice we need at least two control parameters [6]. This is the reflection of the parity symmetry of the model (32).

Choosing $B=\left(\mathbf{b}_{1} \mathbf{b}_{2}\right)$ as a two-column matrix and defining the Fourier coefficients

$$
b_{i}^{k}=\mathbf{e}^{k} \cdot \mathbf{b}_{i}
$$

we write the conditions on the vectors $\mathbf{b}_{1}$ and $\mathbf{b}_{2}: b_{1}^{k}$ $\neq 0, b_{2}^{k} \neq 0$, and $b_{1}^{k} \neq b_{2}^{k}$ for $0<|k|<\pi$, and either $b_{1}^{k} \neq 0$ or $b_{2}^{k} \neq 0$ for $k=0, \pi$. For example, the choice

$$
B_{i j}=\delta_{j, 1} \delta_{i, l}+\delta_{j, 2} \delta_{i, l+1}
$$

yields a controllable system for any $1 \leqslant l \leqslant N$. It corresponds to applying feedback locally through the perturbations of the variables at the adjacent sites $l$ and $l+1$. In fact, it can be easily seen that this control arrangement makes unstable periodic orbit with arbitrary symmetry controllable.

All the examples above show that the symmetry is reduced upon the linearization of the evolution equation. However, the symmetry can increase as well. It is quite easy to construct a coupled map lattice system whose symmetry will increase for certain highly symmetric reference states. We will see another (continuous-time) example just below.

\section{PARTICLE IN A SYMMETRIC POTENTIAL}

The motion of a particle in a symmetric potential

$$
m \partial_{t}^{2} \mathbf{r}=-\nabla V(\mathbf{r})
$$

serves as another example of the relation between the groups $\mathcal{G}$ and $\mathcal{G}^{*}$. Suppose that the potential $V(\mathbf{r})$ possesses the cubic symmetry [group $\mathrm{O} \subset \mathrm{SO}(3)$ ], but is not spherically symmetric, for instance,

$$
V(\mathbf{r})=V_{0} \cosh (k x) \cosh (k y) \cosh (k z) .
$$


Linearizing the evolution equation (40) about the equilibrium point $\mathbf{r}^{*}=\mathbf{0}$ we obtain

$$
\partial_{t}\left(\begin{array}{l}
\mathbf{r} \\
\mathbf{v}
\end{array}\right)=\left(\begin{array}{cc}
0 & 1 \\
\omega^{2} 1 & 0
\end{array}\right)\left(\begin{array}{l}
\mathbf{r} \\
\mathbf{v}
\end{array}\right),
$$

where $\omega^{2}=-V_{0} k^{2} / m$ and $\mathbb{1}$ is a $3 \times 3$ unit matrix. If $V_{0}<0$ the equilibrium is unstable. Equation (42) is spherically symmetric, $\mathcal{G}^{*}=\mathrm{SO}(3)$, and therefore $\mathcal{G} \subset \mathcal{G}^{*}$, i.e., the symmetry of the linearized equation is higher than the symmetry of the original nonlinear evolution equation.

Next we notice that the representation $T$ of $\mathcal{G}^{*}$ in the six-dimensional space $\{\mathbf{r}, \mathbf{v}\}$ can be decomposed into a sum of two three-dimensional irreducible representations of $\mathrm{SO}(3)$ (vector representations):

$$
T=T^{1} \oplus T^{1}, \quad m_{1}=3 .
$$

This indicates that in order to control the unstable state $\mathbf{r}^{*}$ $=\mathbf{v}^{*}=0$ one needs at least three independent control parameters.

Probably the simplest way to control such a system is to readjust the potential (applying external fields, shifting support point, etc.) based on the instantaneous values of the position $\mathbf{r}$ and velocity $\mathbf{v}$ of the particle. This corresponds to picking the control matrix in the form

$$
B=\left(\begin{array}{ccc}
0 & 0 & 0 \\
\mathbf{b}_{1} & \mathbf{b}_{2} & \mathbf{b}_{3}
\end{array}\right),
$$

where $\mathbf{b}_{1}, \mathbf{b}_{2}, \mathbf{b}_{3}$ could be chosen as any three linearly independent vectors in $R^{3}$.

\section{CONCLUSIONS}

Summing up, we conclude that the symmetry properties of the system should be understood prior to constructing a control scheme. The number of control parameters required to control a given state of the system can often be determined using only symmetry considerations, without knowing anything else about the actual evolution equations. The knowledge of the evolution equations (at least in the linearized form), however allows one to choose the control parameters (through the matrix $B$ ) systematically, avoiding a trial-anderror search. The general idea can be stated briefly: The controllability condition requires the control arrangement able to break the symmetry of the evolution equation completely.

Care should be taken if there are accidental degeneracies. The knowledge of the multiplicity of the degenerate eigenvalues becomes less useful and typically leads to an overestimation of the number of control parameters required. This case is more complicated and additional information about the structure of the Jacobian might be necessary in order to determine the minimal number of control parameters and construct the control matrix.

\section{ACKNOWLEDGMENTS}

R.O.G is grateful to J. C. Doyle and R. Murray for valuable discussions. This work was partially supported by the NSF through Grant No. DMR-9013984.
[1] F. J. Romeiras, C. Grebogi, E. Ott, and W. P. Dayawansa, Physica D 58, 165 (1992).

[2] V. Petrov, E. Mihaliuk, S. K. Scott, and K. Showalter, Phys. Rev. E 51, 3988 (1995).

[3] M. Ding, W. Yang, V. In, W. L. Ditto, M. L. Spano, and B. Gluckman, Phys. Rev. E 53, 4334 (1996).

[4] E. Barreto and C. Grebogi, Phys. Rev. E 52, 3553 (1995).

[5] J. Warncke, M. Bauer, and W. Martienssen, Europhys. Lett.
25, 323 (1994).

[6] R. O. Grigoriev, M. C. Cross, and H. G. Schuster, Phys. Rev. Lett. 79, 2795 (1997).

[7] M. Locher and E. R. Hunt, Phys. Rev. Lett. 79, 63 (1997).

[8] J. E. Rubio, The Theory of Linear Systems (Academic, New York, 1971).

[9] M. Hammermesh, Group Theory and Its Application to Physical Problems (Addison-Wesley, Reading, MA, 1964). 